\title{
Tabularia
}

TABULARIA Sources écrites des mondes normands médiévaux Guillaume de Volpiano : Fécamp et l'histoire normande | 2002

\section{Dudon de Saint-Quentin et Fécamp}

Dudo of Saint-Quentin and Fécamp

\section{Pierre Bouet}

\section{(2) OpenEdition}

\section{Journals}

Édition électronique

URL : http://journals.openedition.org/tabularia/1774

DOI : 10.4000/tabularia. 1774

ISSN : 1630-7364

Éditeur :

CRAHAM - Centre Michel de Boüard, Presses universitaires de Caen

Référence électronique

Pierre Bouet, « Dudon de Saint-Quentin et Fécamp », Tabularia [En ligne], Guillaume de Volpiano Fécamp et l'histoire normande, mis en ligne le 25 octobre 2002, consulté le 01 mai 2019. URL : http:// journals.openedition.org/tabularia/1774; DOI : 10.4000/tabularia.1774 


\title{
Dudon de Saint-Quentin et Fécamp Dudo of Saint-Quentin and Fécamp
}

\author{
Pierre BOUET \\ Université de Caen Basse-Normandie \\ p.bouet@mrsh.unicaen.fr
}

Résumé:

La ville de Fécamp fut le lieu de naissance et de sépulture du duc Richard I ${ }^{\text {er }}$ qui y fit édifier une collégiale, dédicacée en 990. Dudon de Saint-Quentin, historiographe des premiers ducs de Normandie, parle peu de cette ville: il évoque cependant le rôle important que celle-ci a joué durant le $\mathrm{X}^{\mathrm{e}}$ siècle au temps de Guillaume Longue Epée et de Richard I ${ }^{\text {er }}$ (c. 927-996). Les fouilles d'Annie Renoux ont confirmé l'existence d'une résidence ducale (avec aula, camera et capella), modeste au temps de Guillaume Longue Epée, plus importante sous le règne de Richard I ${ }^{\text {er }}$. Dudon a, sans doute, voulu dans les dernières pages de sa chronique promouvoir un culte en l'honneur du duc Richard, mort en «odeur de sainteté » dans la cité de Fécamp et inhumé dans la collégiale qu'il avait fait construire.

Mots-clés: Fécamp, Dudon de Saint-Quentin, Guillaume Longue Epée, Richard I ${ }^{\text {er }}$, historiographie, résidence ducale.

\begin{abstract}
:
The town of Fécamp was the place of birth and death of Duke Richard I who had a collegiate church built there, which was consecrated in 990. Dudo of Saint-Quentin, the historiographer of the first dukes of Normandy, provides little information about the town; he mentions however its major role in the tenth century under William Longsword and Richard I (c. 927-996). Annie Renoux's excavations confirmed the existence of a ducal residence (with aula, camera and capella), which was relatively modest under William Longsword, but more opulent under Richard I. In the last pages of his chronicle, Dudo probably wanted to exhort to the worship of Duke Richard, who died "in the odour of sanctity" in the town of Fécamp and was buried in the collegiate church he had built.
\end{abstract}

Keywords: Fécamp, Dudo of Saint-Quentin, William Longsword, Richard I, historiography, ducal residence. 
Dudon, chanoine de Saint-Quentin, composa dans les dernières années du $\mathrm{X}^{\mathrm{e}}$ siècle un ouvrage volumineux traditionnellement intitulé De moribus et actis primorum Normanniae ducum. Ce titre qui s'inspire d'un passage de la préface dédicatoire adressée à Adalbéron, évêque de Laon ${ }^{1}$, fut imposé par André Duchesne, puis par Jules Lair, les deux éditeurs de Dudon ${ }^{2}$. Comme l'a suggéré Gerda Huisman, il conviendrait mieux de retenir l'intitulé Gesta Normannorum, voire Historia Normannorum, puisque ce sont ces dénominations que l'on rencontre sur de nombreux manuscrits ${ }^{3}$.

Dudon offre dans ses quatre livres un panorama de l'histoire de la Normandie $\mathrm{du} \mathrm{X}^{\mathrm{e}}$ siècle en centrant son récit sur les figures les plus marquantes. Le livre I est consacré à Hasting, le chef viking, particulièrement cruel et barbare, incarnation parfaite du pirate viking capable des méfaits les plus sanguinaires. Le livre II narre les exploits de Rollon à qui la Providence a confié la mission de rétablir la paix dans l'Occident carolingien et de devenir le nouveau protecteur de l'Eglise, pour prix de sa conversion. Les livres III et IV rapportent les faits essentiels, ou jugés tels par Dudon, des règnes de Guillaume Longue Epée (c. 927-942) et de Richard I ${ }^{\text {er }}$ (942-996).

Cet ouvrage est vite devenu une référence pour les historiens médiévaux et normands en particulier. Il était lu et copié, même après 1070 quand Guillaume de Jumièges en eut publié un abrégé avec une continuation pour l'histoire des ducs Richard II et Richard III, Robert le Magnifique et Guillaume le Bâtard. Il nous reste actuellement quatorze manuscrits dont quatre du XI ${ }^{e}$ siècle. Le plus ancien manuscrit (Berne, Burgerbibliothek Bongars 390) date de la première moitié du XI ${ }^{e}$ siècle et ne contient que le texte en prose, sans la préface et les nombreux poèmes qui interrompent le récit aux moments forts de la narration. Les trois autres, qui furent copiés au cours de la seconde moitié du XI ${ }^{e}$ siècle, présentent un texte complet avec préface et poèmes: le manuscrit de la bibliothèque municipale de Rouen Y 11 qui fut copié à Jumièges, celui de Cambridge (Corpus Christi College 276), attribué au scriptorium de l'abbaye Saint-Augustin de Cantorbéry et le manuscrit de Berlin (Deutsche Staatsbibliothek Phillipps 1854) qui appartint à La Trinité de Fécamp. Ce dernier manuscrit, qui fit partie de la bibliothèque de sir Thomas Phillipps, avait été consulté par A. Duchesne et J. Lair pour leurs éditions : il présente la particularité de posséder un poème final à la gloire de Fécamp et de l'abbé Jean de Ravenne. Selon Jonathan James Alexander, ce manuscrit aurait été copié au scriptorium du Mont-Saint-Michel vers 1050-1070 et offert à Fécamp, à moins qu'il n'ait été prêté à l'abbaye fécampoise et jamais rendu 4 .

1. Dudon DE SAINT-QUentin, De moribus et actis primorum Normanniae ducum, éd. Jules LAIR, Caen, Le Blanc-Hardel, 1865 (M.S.A.N., t. XXIII), p. 115 - 301 ; cf. Préface, p. 119, 7, ligne 8.

2. Duchesne, André, Historiae Normannorum scriptores antiqui, Paris, Lutetiae Parisiorum, 1619, p. 51-160.

3. Huisman, Gerda C., "Notes on the manuscript Tradition of Dudo of Saint-Quentin's Gesta Normannorum », Anglo-Norman Studies, 6, 1983, Woodbridge, Boydell Press, 1984, p. 122-135.

4. AlEXANDER, Jonathan James, Norman Illumination at Mont St. Michel 966 - 1100, Oxford, Clarendon Press, 1970, p. 127- 172 . 
Six autres manuscrits datent du XII ${ }^{\mathrm{e}}$ siècle, dont le manuscrit de Paris (BnF, nouvelle acquisition latine 1031) en provenance de Saint-Wandrille. Au vu des manuscrits existants, on constate que les grandes abbayes bénédictines de Normandie disposaient de l'ouvrage de Dudon dans leur bibliothèque: Jumièges (ms de Rouen), Saint-Wandrille (ms de Paris), Fécamp / Mont-Saint-Michel (ms de Berlin) et SaintEvroult avec le manuscrit 20 de la bibliothèque municipale d'Alençon, disparu depuis l'édition de J. Lair.

La cité de Fécamp occupe peu de place dans l'œuvre de Dudon. C’est dans les dernières pages que l'historien des ducs de Normandie l'évoque. Il convient de voir quelle place tient Fécamp et son abbatiale dans l'économie d'une œuvre au service du lignage de Rollon et de ses descendants. A l'évidence, Dudon connaît bien la région puisqu'il accompagna les ducs Richard ${ }^{\text {er }}$ et Richard II dans leurs différents lieux de pouvoir. Dans deux chartes, en effet, Dudon s'intitule capellanus et cancellarius du duc de Normandie ${ }^{5}$. On peut légitimement penser qu'il participa aux offices des chanoines de La Trinité de Fécamp, puis à ceux des moines bénédictins installés vers 1001 par Guillaume de Volpiano.

Le premier événement relatif à Fécamp dont Dudon nous parle concerne la naissance du jeune Richard ${ }^{\text {er }}$ vers 932. L'historien mentionne la cité de Fécamp au livre III, consacré à Guillaume Longue Epée, après avoir raconté la défaite du rebelle Riouf à l'issue d'un combat livré au Pré de la Bataille près de Rouen : «Alors que Guillaume revenait du combat, arriva de Fécamp un chevalier qui lui annonça que sa très chère épouse lui avait donné un fils. Plus heureux de la naissance de son fils que de sa victoire, il y envoya Henri, l'évêque de Bayeux, le plus vénérable de tous les prélats, et Bothon, le plus éminent de ses chevaliers, pour que son fils renaisse et soit renouvelé par l'huile, par le chrême et par l'eau baptismale » ${ }^{6}$.

Ces quelques lignes nous fournissent des informations importantes sur Fécamp vers 930-935. Il semble donc qu’à cette époque Guillaume Longue Epée ait possédé à Fécamp une résidence susceptible d'accueillir l'épouse du duc avec sa domesticité. Guillaume de Jumièges qui résume Dudon en apportant souvent corrections et additions et qui est très bien informé dit même qu'il y avait un castrum (c'est-à-dire

5. FaurouX, Marie, Recueil des Actes des ducs de Normandie de 911 à 1066, Caen, Caron, 1961 (M.S.A.N., t. XXXVI) : $\mathrm{n}^{\circ} 13$, p. 89 : Dudo capellanus Richardi Northmannorum ducis et marchionis hanc cartam composuit et scripsit.; $\mathrm{n}^{\circ} 18$, p. 102 : Dudo cancellarius scripsit et subscripsit.

6. DudON..., 3, 46, p. 191, lignes 10-15: Reuertenti igitur Willelmo de praelio, occurrit ei miles quidam ex Fiscanno, nuntians quod esset ei filius ex coniuge dilectissima natus. Laetior itaque peracto praelio, laetissimusque filio, misit Henricum, Baiocensis ecclesiae episcopum, omnium quippe praesulum sanctissimum, et Bothonem, cunctorum militum praecellentissimum, sacri baptismatis rore oleo et chrismate renasci et innouari filium. 
une place forte) avec une garnison sous les ordres d'un praefectus ${ }^{7}$. En outre, c'est bien à Fécamp que le duc Guillaume envoie Henri et Bothon : à cette époque, en effet, les évêques des diocèses les plus occidentaux de la province demeuraient le plus souvent à Rouen, à proximité de la cour ducale. C'est par une erreur de lecture que Guillaume de Jumièges a affirmé que le duc Guillaume envoya son fils nouveau-né à Bayeux auprès de l'évêque Henri pour qu'il soit baptisé ${ }^{8}$ : au lieu de lire misit Henricum ( «il envoya Henri»), Guillaume de Jumièges a compris misit ad Henricum ( «il envoya auprès d'Henri »). La tradition manuscrite confirme bien misit Henricum, ce qui laisse supposer que Fécamp possédait déjà un édifice religieux (chapelle, oratoire, église) susceptible d'accueillir la cour ducale pour une grande cérémonie.

Dans le livre IV consacré à Richard ${ }^{\mathrm{er}}$, Dudon parle à nouveau plus longuement de la naissance du jeune Richard en apportant d'intéressantes informations sur sa résidence fécampoise. Il donne en particulier les raisons qui ont amené Guillaume Longue Epée à confier à la cité de Fécamp le destin de sa jeune femme et de son fils : «Il fit transporter son épouse enceinte... avec une importante escorte de chevaliers à sa résidence de Fécamp, de sorte que, si par hasard Riouf, la plus cruelle de toutes les bêtes sauvages, parvenait à s'approprier avec ses complices le pouvoir sur la région normande, comme cela lui semblait probable, son épouse puisse rapidement gagner l'Angleterre pour échapper à la capture ${ }^{9}$. Ce choix d'une cité de bord de mer, avec un site naturel aisé à défendre, comme ultime refuge en cas de perte du pouvoir, apparaît tout à fait vraisemblable. Ces remarques de Dudon confirment d'autres passages du De moribus et actis où se révèle la fragilité du pouvoir instauré par Rollon et ses descendants. Dudon présente, d'ailleurs, la révolte de Riouf comme le refus des Vikings de la Normandie occidentale à accepter l'autorité d'un prince déjà bien intégré à la culture chrétienne d'Occident. Mais cette menace n'est pas la seule. A ce danger intérieur s'ajoute la menace (réelle ou supposée) des attaques lancées par les comtes des régions limitrophes. Dans ce contexte politique la cité de Fécamp offrait une garantie défensive et une situation excentrée que la capitale de Rouen, même avec ses hautes murailles, ne pouvait lui offrir. La mise en défense d'Eu, d'Arques et de Fécamp semble être un élément du dispositif de gouvernement dont la vocation n'était pas seulement d'arrêter le débarquement d'autres bandes vikings. $\mathrm{Si}$, comme le dit Dudon dans le poème qui suit, « Richard a par sa naissance honoré

7. Guillaume de Jumièges, Gesta Normannorum ducum, éd. Elisabeth Van Houts, Oxford, Clarendon Press, (Oxford Medieval Texts), 1992, 3, 2, p. 78 : ... a prefecto Fiscannensis castri legatus dirigitur...

8. Ibidem, 3, 2, p. 78: Qui, letus ualde effectus, sub festinatione Baiocas illum episcopo Henrico mandauit dirigere...

9. DUdON..., 4, 66, p. 218, lignes 7-11:... (Willelmus) transuehi fecit decenti insigniter equitatu ad Fiscannicae sedis aulam, ut si forte Riulfus, omnium belluarum crudelissimus, Northmannicae regionis monarchiam cum suis complicibus sibi uindicaret, ut aestimabatur, ne eam raperet, ad Anglos citius transfretaretur. 
la cité de Fécamp ${ }^{10}$, c'est parce que Fécamp garantissait au lignage de Rollon sa survie et son maintien au pouvoir.

Dudon raconte en détail les cérémonies du baptême du jeune Richard en présence de tous les évêques de la région, du clergé et d'un peuple en liesse : l'enfant fut donc baptisé par une triple immersion (trinae immersionis inundatione) ${ }^{11}$ au nom de la sainte Trinité et reçut le nom de Richard.

Les termes utilisés par l'historien des ducs méritent une analyse linguistique. A propos de Fécamp, Dudon parle d'un castrum, d'un oppidum, de moenia et d'aula sedis (cf. tableau des termes employés par Dudon pour Fécamp et les autres sites de Normandie). Ces termes n'ont pas la précision sémantique que nous aimerions rencontrer: le souci littéraire et artistique l'emporte toujours sur l'exigence historique aussi bien dans l'historiographie antique que dans l'historiographie médiévale. Un même édifice, comme la cathédrale de Rouen, peut être qualifiée à quelques lignes d'intervalle d'ecclesia, de basilica, de templum, de delubrum, voire de monasterium. Cela ne veut pourtant pas dire que la terminologie de Dudon est floue et sans rigueur. Les connotations contextuelles permettent très souvent de définir le sens précis des termes employés ${ }^{12}$.

Dudon distingue ainsi les moenia de Fécamp des moenia d'autres sites: à Fécamp, ce sont des moenia castri, tandis qu'à Rouen, Bayeux, Evreux, qui sont des ciuitates, ce sont des moenia urbis, expression qui évoque des remparts dont certaines parties peuvent remonter aux murailles gallo-romaines. Comme l'a suggéré Annie Renoux dans sa thèse, cette enceinte primitive de Fécamp était une levée de terre (agger) avec fossé et palissade en bois ${ }^{13}$. Dudon ne parle à propos de Fécamp ni d'arx ni de turris, comme c'est le cas pour le château de Montreuil ${ }^{14}$. Fécamp appartient donc à ce type de places fortes établies dans la campagne en s'appuyant sur des sites naturellement bien défendus: les termes castrum et oppidum dont use Dudon pour Fécamp correspondent à une plate-forme entourée d'une enceinte de terre vraisemblablement renforcée par un fossé et une palissade à l'intérieur de laquelle sont édifiés divers bâtiments. En dehors d'Evreux, Dudon réserve l'appellation de castrum à des places de campagne.

10. Ibidem, Apostropha, p. 220, v. 11-14: Viri nomine perpeti / Castro facta decentius / O Fiscanne tuo, maris / Prope littora quod uiget.

11. Ibidem, 4, 66, p. 219, ligne 39.

12. Cf. au livre IV, 126, p. 290-291, Dudon use des termes suivants : ecclesias, templa, monasterium (cathédrale de Rouen), ecclesia (monastère Saint-Ouen de Rouen), delubrum (monastère du Mont Saint-Michel), basilica / delubrum / templum (pour la nouvelle construction de Fécamp).

13. RENOUX, Annie, Fécamp: du palais ducal au palais de Dieu, Paris, Editions du CNRS, 1991, p. 314317 ; Idem, "Châteaux normands du $\mathrm{X}^{\mathrm{e}}$ siècle dans le De moribus et actis primorum Normanniae ducum de Dudon de Saint-Quentin ", Mélanges d'archéologie et d'histoire médiévales en l'honneur du Doyen Michel de Boüard, Genève / Paris, Droz, 1982, p. 327-346 et notamment p. 329-337.

14. Dudon..., 3, 60, p. 205, ligne 17 : ... inexpugnabilium praesidio turrium munitum castrum... 
Dans ce castrum s'élève une aula qui fait référence à une résidence princière d'une certaine importance puisque c'est le terme réservé aux résidences du roi de France, de l'empereur Othon, du comte de Poitiers. Qu'il y ait dans l'emploi de ce terme la volonté de valoriser la cour normande, c'est vraisemblable, mais le fait incontournable est que Fécamp est capable d'accueillir la cour ducale pour une cérémonie d'une certaine importance : cela suppose l'existence d'un complexe résidentiel avec une grande salle de réception, des bâtiments privés et une chapelle. Les fouilles archéologiques d'Annie Renoux ont découvert la trace d'un bâtiment résidentiel en bois de 16,5 m de long sur 5,5 m de large divisé en deux salles, selon la tradition des résidences aristocratiques de l'époque carolingienne ${ }^{15}$. Une chronique contemporaine de l'ouvrage de Dudon, le manuscrit 528 de la bibliothèque municipale de Rouen, parle même d'un palatium mirifico opere que Guillaume Longue Epée fit construire à Fécamp, à proximité de l'ancien monastère détruit où les arbres d'une vaste forêt avaient remplacé les moniales. Ainsi, le texte de Dudon qui repose sur les informations de Raoul d'Ivry, le demi-frère de Richard I ${ }^{\text {er }}$, établit clairement que Fécamp fut sous le règne de Guillaume Longue Epée une place forte, un castrum-oppidum, située près de la mer: Fécamp n'est ni une ciuitas, ni une urbs au sens strict, mais une résidence princière, situé à proximité d'un port, qui va devenir un lieu de pouvoir et un refuge pour le duc et sa famille dans la longue et difficile reconnaissance de sa légitimité dynastique.

C'est dans les dernières pages du De moribus et actis que Dudon parle longuement de Fécamp et de son abbatiale. Le chapitre 126 du livre IV expose de façon synthétique la politique religieuse du duc Richard I ${ }^{\text {er }}$ dans le domaine de l'architecture. Richard restaura ou édifia de nombreuses églises tant en Francia qu'en Normandie. Il ne nous énumère pas les lieux où ces édifices furent élevés, il n'évoque que les plus prestigieux d'entre eux : la cathédrale de Rouen, le monastère SaintOuen et le Mont Saint-Michel. Alors qu'habituellement Dudon, comme tous les chroniqueurs médiévaux, utilise des expressions toutes faites, du genre delubrum mirae amplitudinis construxit, pour décrire les nouvelles constructions, l'auteur du De moribus et actis consacre un long chapitre à l'histoire de la construction de l'abbatiale de Fécamp et à la description minutieuse de son architecture, de son décor et de son trésor. Pourquoi deux lignes sur la cathédrale de Rouen où étaient inhumés les deux premiers ducs, Rollon et Guillaume Longue Epée, et deux pages sur Fécamp?

Ce chapitre doit être remis en perspective avec le récit de la mort et des funérailles du duc Richard I ${ }^{\text {er }}$. Les derniers chapitres et le poème à la gloire de Fécamp constitu-

15. Renoux, Annie, Fécamp..., p. 367-375. 
ent l'épilogue du projet historiographique de Dudon de Saint-Quentin ${ }^{16}$. Celui-ci a pris la plume à la fin du $\mathrm{X}^{\mathrm{e}}$ siècle pour affirmer la légitimité du lignage de Rollon à exercer le pouvoir sur tout le duché de Normandie. Cette légitimité a, selon Dudon, des fondements multiples. Elle a, d'abord, un fondement de nature politique dans la mesure où c'est Rollon qui fut le négociateur de Saint-Clair-sur-Epte et que c'est lui qui a conclu le traité au terme d'un rapport de forces dont il est sorti vainqueur. La légitimité des Rollonides a également un fondement religieux, puisque c'est Rollon qui, comme son ancêtre Enée, a reçu la mission providentielle de rétablir la paix dans le royaume de Francia et de restaurer le culte chrétien pour prix de sa conversion ${ }^{17}$. Le discours, qui s'exprime de façon rhétorique ou poétique à travers tout le De moribus et actis, s'adressait d'abord aux Normands rebelles qui contestaient encore en 996 le pouvoir des Rollonides. A la fin du $\mathrm{X}^{\mathrm{e}}$ siècle, les règles de succession restaient encore, comme dans les autres royaumes et provinces d'Occident, très imprécises et sujettes à contestation. Dudon, qui avait arrêté d'écrire en raison de la mort du duc Richard Ier, se remit au travail à la demande de son fils Richard II : on entrevoit tout le parti que put en tirer le jeune duc pour asseoir son autorité. Le discours de Dudon s'adressait également à l'opinion publique européenne, c'est-à-dire aux cours princières et royales, pour montrer comment les Normands, convertis à la foi chrétienne, étaient devenus des protecteurs et des défenseurs de l'Eglise.

Mais la légitimité des Rollonides s'est affirmée d'une autre façon: Dudon a voulu montrer l'ascension spirituelle du lignage issu de Rollon et même la sainteté de son dernier représentant. C'est pour cela que l'historien panégyriste se change à la fin du livre IV en hagiographe. L'affirmation progressive du pouvoir politique des ducs de Normandie s'est, en effet, doublée d'une évolution intérieure dont chaque duc marque une étape. Rollon est le modèle du prince fondateur qui, converti à la foi chrétienne, use de la force pour imposer la paix et l'ordre dans sa province. Il marque la rupture avec les mœurs barbares et païennes, incarnées par Hasting, le nequissimorum nequior. Son fils Guillaume Longue Epée est le prince de la paix usant, non plus de la force comme son père, mais de la parole. C'est pour rétablir la paix par la négociation qu'il meurt assassiné en martyr, selon le modèle des saints rois subissant la mort dans l'exercice de leur ministerium au service du peuple chrétien. Richard I ${ }^{\text {er }}$, au terme de cinquante quatre années de règne, achève l'œuvre de ses ancêtres: il incarne non plus le saint roi martyr, mais le saint roi confesseur qui, par la pratique des vertus chrétiennes, est parvenu à accomplir parfaitement les tâches de son ministère : maintenir la paix, assurer la justice et protéger l'Eglise. «C'est donc avec

16. Le MaHO, Jacques, «La mort et les funérailles de Richard I Ir duc de Normandie d'après Dudon de Saint-Quentin ", La Normandie vers l'An Mil, Rouen, Société de l'histoire de Normandie, 2000, p. 84-87; BOUET, Pierre, «Dudon de Saint-Quentin: la construction de la nouvelle collégiale de Fécamp (990) », La Normandie vers l'An Mil, p. 123-129.

17. BouET, Pierre, «Dudon de Saint-Quentin et Virgile: L'Enéide au service de la cause normande», Recueil d'études en hommage à Lucien Musset, Caen, Cahier des Annales de Normandie, n’23, 1990, p. 215-236. 
raison, écrit Dudon au chapitre 127, et de façon justifiée que nous déclarons, au vu de ses actions, Richard, le duc de la terre normande, à la fois saint et sacré: car tous les dons des béatitudes évangéliques s'appliquent parfaitement à sa personne ${ }^{18}$. A la fin du $\mathrm{X}^{\mathrm{e}}$ siècle cet idéal du saint roi confesseur s'impose, notamment dans l'empire ottonien avec la canonisation d'empereurs et d'impératrices ${ }^{19}$.

La sainteté du duc Richard devait nécessairement s'incarner dans un lieu sacré: ce lieu sacré, c'est à la fois Richard et la Providence divine qui l'ont choisi. Richard a voulu que le lieu de sa naissance sur la terre soit également celui de son dies natalis dans les cieux. Il avait fait déjà tailler un sarcophage à Fécamp. Lorsqu'il se sentit faiblir, il quitta Bayeux pour se rendre à Fécamp et y vivre ses derniers instants. C'est donc dans la ville où il était né qu'il assura la continuité dynastique en désignant comme son successeur légitime son fils qui portait le même nom. C'est à Fécamp qu'il effectua ses dernières largesses à l'égard de Dieu et des pauvres ${ }^{20}$. C'est à La Trinité de Fécamp, dont il était le fondateur, qu'il voulut reposer, non à l'intérieur de l'église, mais "près de la sortie sous la gouttière $»^{21}$. Ainsi, cité dynastique, Fécamp était choisie comme lieu de sépulture et mausolée du duc de Normandie. Tel est le destin que Richard avait choisi pour la cité de sa naissance. Il y avait fait construire un palatium, un ensemble résidentiel avec fonctions administrative, politique et religieuse. Les fouilles d'Annie Renoux ont mis en évidence des constructions des années 975-980 comprenant un édifice en $\mathrm{L}$ de $143 \mathrm{~m}^{2}$ avec aula à l'étage et une petite construction de $30 \mathrm{~m}^{2}$ situé dans la cour qui devait servir de camera ${ }^{22}$. Quelques années après, il fit édifier la nouvelle collégiale, dédicacée en 990, dont le luxe et les dimensions révèlent la volonté ducale de faire de Fécamp une cité dynastique ${ }^{23}$. Par sa longueur (estimée à 60-65 m) l'abbatiale de Fécamp dotée d'un chœur oriental et d'un massif occidental rivalisait avec les plus grands chefs-d'œuvre de cette fin du X $X^{\mathrm{e}}$ siècle : la Basse CEuvre de Beauvais et Cluny II.

Mais l'historien - hagiographe transcende les projets du duc Richard. Il cherche, en effet, à faire de ce mausolée dynastique un lieu de pèlerinage à la gloire des

18. DudON..., 4, 127, p. 293, lignes 1-3: Merito igitur iusteque atque probabiliter Ricardum Northmannicae regionis ducem beatum sanctumque, recensitis breuiter operibus eius, dicimus; cui omnia euangelicarum beatitudinum dona reperiuntur propensius attributa...

19. Cf. CORBET, Patrick, Les saints ottoniens. Sainteté dynastique, sainteté royale et sainteté féminine autour de l'an mil, Sigmaringen, J. Thorbecke, 1986, 288 p.

20. DudON..., 4, 127, p. 293, lignes 1-3.

21. Ibidem, 4, 128, p. 297, lignes 29-30: Cadauer tanti sceleris non requiescat infra aditum huius templi, sed ad istud ostium in stillicidio monasterii.

22. RenouX, Annie, Fécamp..., p. 375 -387.

23. Richard ${ }^{\text {er }}$ ne fut pas le premier occupant de ce mausolée. Selon Lucien Musset, un fils de Richard ${ }^{\text {Ir }}$, Robert, décéda le lendemain de son baptême et fut inhumé à Fécamp ; cf. MUSSET, Lucien, «Les sépultures des Souverains normands: un aspect de l'idéologie du Pouvoir » in MUSSET, Lucien, BOUVRIS, Jean-Michel et MAILLEFER, Jean-Marie, Autour du pouvoir ducal normand ( $X^{e}-X I^{e}$ siècles), 1985, Cahier des Annales de Normandie, n 17, Caen, p. 30. 
Rollonides. Après avoir montré combien, par ses actions, le duc Richard avait accompli toutes les prescriptions évangéliques, il fait un récit circonstancié des derniers instants du duc où se révèle sa sainteté. Alors que les habitants de Fécamp pleurent déjà la mort imminente du duc, celui-ci affronte les ultimes souffrances avec lucidité: revêtu du cilice, il a la force d'aller nu-pieds à l'autel de la collégiale de La Trinité pour y prier et y présenter ses derniers dons. Dudon, à la manière des hagiographes, décrit les épreuves ultimes parfaitement assumées: paralysie des pieds, des jambes, puis de tous les membres. Au dernier instant le duc leva les yeux et les mains vers le ciel et dit : «Entre tes mains, Christ, je remets mon esprit ». ${ }^{24}$ Ainsi mourut le duc Richard, en prononçant les paroles mêmes que Jésus prononça sur la Croix à l'instant de sa mort. Quand le lendemain on ouvrit le sarcophage, le corps du duc « ressemblait à celui d'un homme vivant » (inuenit omnia eius membra quasi uiui hominis) et du sarcophage s'éleva « une suave odeur de térébenthine et de baume» (odor suauior fragrantia terebenthinae et balsami) ${ }^{25}$, marque objective de la sainteté du défunt.

Ainsi Fécamp devient pour Dudon la cité honorée par la sainteté de son duc. Comme il le proclame dans la poésie finale: Fécamp conserve son corps sacro-saint qui va purifier la cité de ses fautes et la faire prospérer. C'est sur un ton prophétique qu'éclate cet éloge de Fécamp: «Ô Fécamp, toi qui conserves au sein de la terre sacrée les restes des saints qui ont brillé par leurs mérites, tu es toute resplendissante ${ }^{26}$. Dudon évoque alors deux autres figures illustres qui ont rendu célèbre la ville de Fécamp : avant Richard, il y eut saint Léger, qui, privé de la vue et de la langue, trouva le salut à Fécamp. Il y eut également la fondatrice des moniales du VII ${ }^{\mathrm{e}}$ siècle que Dudon nomme par erreur Gildeberta au lieu de Hildemarca. Le corps du duc qui repose en «terre consacrée» (terrae sacratae $)^{27}$ de Fécamp devient dès lors un gage de salut et de prospérité pour la cité: «par ses prières tu sera purifiée de toute souillure, par ses mérites tu parviendras au salut ${ }^{28}$. Puisque le duc Richard a été autrefois « un soutien efficace » (Et cuius patrocinio suffulta fuisti), il sera désormais «le garant de son élévation » (Munere largifluo et cuius praecelsa uigebis) ${ }^{29}$. Ainsi le destin temporel et spirituel de la cité repose-t-il sur la présence agissante du duc, tant après sa mort que de son vivant.

24. Dudon..., 4, 128, p. 297, lignes 33-34: In manus tuas, Christe, commendo spiritum meum.

25. Ibidem, 4, 129, p. 299, lignes 34-35.

26. Ibidem, 4, 129, p. 299: v. 1-3: O Fiscanne, sacris semper fecunde fauillis / Sanctorum cineres meritorum flore micantes / In gremio terrae conseruans iamque sacratae.

27. Ibidem, v. 3.

28. Ibidem, v. 22-23: Cuius et precibus lue purificaberis omni / Et cuius meritis migrabis ad aethera dignis...

29. Ibidem, v. 25-26 
Il est surprenant de constater que Dudon n'a pas fait la moindre allusion à Guillaume de Volpiano qui vint à la demande du duc Richard II établir en 1001 une communauté de moines bénédictins. Ce silence est-il la réponse du chanoine Dudon supportant difficilement l'expulsion des chanoines fécampois? Cela est peu vraisemblable.

La raison de cette absence doit être recherchée ailleurs. Le De moribus et actis s'achevait avec la mort exemplaire de Richard ${ }^{\text {er }}$ en 996. Dudon n'est pas un annaliste qui peut toujours ajouter un fait nouveau à un autre fait. Parler de Guillaume de Volpiano, c'était déjà évoquer l'action politique et religieuse de Richard II. C'était un autre sujet, une autre histoire. Dudon est un historien qui détermine précisément l'objet de sa narration et qui s'attache à la cohérence de son projet historiographique: il avait voulu montré l'élévation d'un lignage qui, plongé dans la barbarie du paganisme, avait, en se convertissant à la foi chrétienne, gravi les honneurs des grandeurs temporelles et atteint les sommets de l'ordre spirituel. La mort de Richard $I^{\text {er }}$ en fut, en quelque sorte, l'apothéose.

Une autre raison tient sans doute au fait que ce projet fut mené à son terme peu de temps après le décès de Richard I ${ }^{\text {er }}$. Que Dudon se soit arrêté d'écrire pour faire son deuil, comme il nous le confie dans sa préface, cela ne saurait se compter en de nombreuses années, d'autant que l'arrivée au pouvoir de Richard II rendait indispensable le soutien précieux que pouvait apporter l'historien en réaffirmant la légitimité du lignage de Rollon. Vers l'an mil, le livre était sans doute déjà achevé : la profonde unité de style et de ton milite en faveur d'une rédaction sans longue interruption. Peut-être apporta-t-il par la suite quelques compléments avec la préface et les poèmes. L'existence de manuscrits ne présentant que le texte en prose semble le confirmer. Le plus ancien manuscrit du De moribus et actis, qui date du début du $\mathrm{XI}^{\mathrm{e}}$ siècle, le Burgerbibliothek de Berne, qui ne contient que le texte en prose, en serait peut-être un témoignage. Des marques effectuées par le copiste aux endroits où les poèmes devaient être insérés, en vue de procéder à une nouvelle copie qui allait comporter le texte complet avec la préface et les poèmes, laissent supposer que l'œuvre en prose dut faire l'objet d'une première publication.

C'est de la même époque que date la Chronique de Fécamp qui se trouve dans le manuscrit 528 de la bibliothèque municipale de Rouen. Comme l'a montré Mathieu Arnoux $^{30}$, ce texte est constitué de plusieurs extraits de vie de saints (Vita sancti Waningi, Vita sancti Leodogarii, Vita sancti Wandregisili) et d'un large extrait de Dudon relatant la construction de l'abbatiale de Fécamp. L'auteur de cette chronique se contente de reprendre mot à mot le texte des sources. Cette brève chronique estelle l'œuvre de Dudon comme le suggère Mathieu Arnoux? C'est peu vraisemblable. Dudon n'a guère l'habitude de recopier fidèlement ses sources: il aime intégrer les

30. ARNOUX, Mathieu, «Before the Gesta Normannorum et beyond Dudo: some evidence on early Norman Historiography", Anglo-Norman Studies, 22, 1999, Woodbridge, Boydell Press, 2000, p. 29-48. L'édition du texte de la Chronique de Fécamp se trouve p. 43-46. 
éléments, empruntés à diverses sources, à son discours rhétorique si particulier. Un examen minutieux des parties rédigées par l'auteur de la chronique, sans tenir compte des emprunts textuels, montre que ce texte est étranger à la plume de Dudon (cf. tableau comparatif des expressions de la chronique du manuscrit 528 avec celles de Dudon). Une grande partie du lexique ne se rencontre jamais chez Dudon : uita decedere, praefatum, non minimum, stragem dare, migrare a saeculo, angelico ductu, indubitatum est, simili modo etc. Le terme Dani, utilisé régulièrement par l'auteur du manuscrit 528, n'est employé qu'une seule fois par Dudon qui préfere le terme Daci (117 occurrences). En outre, les formules identiques que l'on trouve dans la Chronique et chez Dudon ne montre aucun lien de parenté, tant ces expressions sont banales. Mais si le style est fortement différencié, l'intention est bien la même: montrer que Fécamp est non seulement la cité dynastique des ducs de Normandie, mais également une «terre consacrée » par de nombreux miracles prouvant la sollicitude divine.

Tableau des termes employés par Dudon pour Fécamp et pour les autres sites de Normandie

(Les références à Dudon de Saint-Quentin renvoient à l'édition de Jules Lair : livre, chapitre et ligne)

Moenia : 24 occurrences

Fécamp 3 :

Fiscanni castri moenia $(4,66,6)$; moenia Fiscannina $(4,66,33)$; Fiscanninae sedis moenia $(4,126$, 12)

Rouen 8:

moenia urbis Rotomagensis $(3,60,32)$ cf. $3,44,63$; $4,83,1 ; 4,130,20 ; 4,97,15 ; 4,98,12 ; 4,105$, $27 ; 4,116,19$

Bayeux 1:

Baiocensia moenia $(4,68,6)$

Beauvais 1:

moenia urbis Beluacensis $(4,97,8)$

Evreux 1:

Paris 1:

moenia urbis Ebroicacensis $(4,72,14)$

moenia urbis Parisiciae $(4,94,55)$

Mt-St-Michel 1: $\quad$ spatiosa monachilis habitationis moenia $(4,126,9)$

Luna 1 :

per moenia urbis $(1,7,12)$

Crète 1 :

Norvège 1 :

Minoenia moenia $(1,1,9)$

moenia ciuitatis $(2,4,11)$

Généralités 5 :

urbes et moenia restaurare... $(2,27,6) \mathrm{cf} .2,6,32$;

$4,80,10 ; 4,130,23$

Oppidum : 6 occurrences

Fécamp 1:

Fiscanninae sedis oppidum $(4,129,8)$

Tabularia «Études », n 2, 2002, p. 57-70, 25 octobre 2002 
Généralités 5: $\quad$ urbes et castra, uillas et oppida, aulas et palatia (2, $19,17)$

cf. $2,12,7 ; 2,14,17 ; 2,18,13 ; 2,18,22$

Castrum : 44 occurrences

Fécamp 2:

Fiscanni castri moenia $(4,66,6)$; castro facta decentius tuo $(4,66,57)$

Montreuil 16: castrum Monasterioli $(4,59,13)$ cf. 4,59 et $60 ; 4$, 86,$29 ; 4,88,7$ et 14

Coucy 1: Codiciacum castrum $(4,75,14)$

Creil 1:

Cretheltense castrum $(4,77,7)$

Evreux 1:

Ebroicacense castrum $(4,117,10)$

Camp viking 4 : carneum castrum $(2,24,51)$ cf. $2,14,2$ et $7 ; 2,24,45$

Divers 20

Palatium : 6 occurrences

Fécamp 1:

Fiscanni palatio $(4,128,12)$

Rouen 2:

Rotomagensis urbis palatia $(4,72,17)$ et $(4,102$, 17)

Laon 2: $\quad$ palatio regis $(4,74,10)$, palatio meo $(4,72,7)$

Angleterre 1: $\quad$ aulas et palatia $(2,19,17)$

Aula : 13 occurrences

\section{Fécamp 3 :}

Fiscannicae sedis aulam $(4,66,9 ; 4,128,11$ et 23$)$

Roi de France 2:

Francisca aula $(2,5,37)$, aula suae habotationis (4, 70, 45)

Comte de Poitiers 1: $\quad$ Pictauensis aula $(3,47,27)$

Empereur Othon 1: $\quad$ tuae sedis aula $(4,96,60)$

Sens général 2: $\quad$ aulas et palatia $(2,19,17$

Sens métaphorique 4 : coelestis aula (Préf., 7,31 ) 


\section{Comparaison entre Bibl. mun. Rouen ms 528 et Dudon de Saint-Quentin}

\begin{tabular}{|c|c|c|}
\hline Ms 528 & Expressions communes & Ressemblances \\
\hline 1-Illustris prudentia & - & - \\
\hline Eminens bonitate & - & $\begin{array}{l}\text { famosissimus bonitate } 4,113,7 \\
\text { diffusus bonitate } 4,126,62\end{array}$ \\
\hline Deo obediens & - & $\begin{array}{l}\text { obediens domino } 4,95,8 \\
\text { obediens Dei praeceptis } 4,106,9\end{array}$ \\
\hline 3- defuncto rege & $\begin{array}{l}\text { defuncto rege/ illo } 2,2,8 ; \\
3,38,15 ; 4,127,59\end{array}$ & - \\
\hline regnum suscepit & - & $\begin{array}{l}\text { regnum accipere } 2,33,5 \\
\text { regnum obtinere } 3,47,2\end{array}$ \\
\hline regnum tenens & regnum tenere $2,18,18$ etc & - \\
\hline vita decessit & - & - \\
\hline huius tempore & - & - \\
\hline uinculis tenebat & - & - \\
\hline praefatum Waningum & - & - \\
\hline 4-floruit locus & - & - \\
\hline gens saeuissima & - & saeua gentilitas $2,1,99$ \\
\hline Dani & Dani (1emploi : $2,13,14$ ) & Daci (117 emplois) \\
\hline Irrumpo & - & Irruo \\
\hline Ora maritima & - & - \\
\hline Non minimum & - & - \\
\hline Stragem dedit & - & stragem perago $4,112,28$ \\
\hline Factum est ut & factum est ut $4,74,9$ & - \\
\hline Sanctimoniales & - & - \\
\hline Ad solitudinem redigeret & - & - \\
\hline Christocolarum ornamentum & - & - \\
\hline 5- gubernante regnum & - & gubernat populos $4,103,18$ \\
\hline natione Danus & - & natione Graecus 2, 30, 19 \\
\hline ritu gentilis & - & ritu ueterrimo $1,1,26$ \\
\hline ordine miles & - & - \\
\hline intrat Frantiam & intrat Scaldi alueum 2, 9, 35 & - \\
\hline deuastans terram & (3 emplois) $2,9,20 \ldots$ & - \\
\hline baptismi gratiam & - & baptismum suscepit $1,6,4$ \\
\hline quoad & - & - \\
\hline iura et leges paternas & $\begin{array}{l}\text { leges et iura paternaque decreta } \\
3,58,8\end{array}$ & - \\
\hline migrarut a saeculo & $\begin{array}{ll}- \\
-\end{array}$ & $\begin{array}{l}\text { migrare ad Christum } 3,68,7 \\
\text { migrare ad aethera } 3,129,68\end{array}$ \\
\hline $\begin{array}{l}\text { mirifico opere (construxit) } \\
\text { eleganti forma infantem } \\
\text { imbui disciplini }\end{array}$ & $\begin{array}{l}- \\
-\end{array}$ & $\begin{array}{l}\text { miri schematis templum } 3,58 \text {, } \\
10 \\
\text { elegantissimae formae uirgo } 3 \text {, } \\
76,6 \\
\text { imbutus fide } 2,6,19\end{array}$ \\
\hline destructo monasterio & destructa templa 2, 3, 9 & - \\
\hline $\begin{array}{l}\text { grandis silua } \\
\text { ad praesens }\end{array}$ & ad praesens (passim) & - \\
\hline
\end{tabular}




\author{
auctor et redemptor \\ redemptor humani generis \\ 6- Miro opere \\ Ad memoriam (confessoris) \\ (ostendere) mortalibus \\ conditoris iussa \\ obediens iussis \\ angelico ductu \\ (sine) adiutorio humano \\ 7- Circumhabitantes \\ Officium diuinum \\ Uir decorus \\ Omnibus uidentibus \\ oratorium (ingressus) \\ superaltare posuit \\ nulli nichil \\ indubitatum (est) \\ pro magno opere \\ in testimonio \\ 8-Pre memorie Ricardus
}

in regnum succedere

9- Uir bonae uitae

missarum sollemnia
more solito
panem dominicum
simili modo
peractis mysteriis
quae acciderant exposuit
repletus gaudio
gratias agit
congregata multitudine
dux memoratus
conuocatis episcopis
cum magno gaudio
in donum dedit

redemptor humani generis 4 , plasmator et redemptor 4, 126, 126,17 17

obediens praeceptis Dei $4,106,9$ obediens praeceptis Rollonis 2, 9,32

officium diuinum 4, 115, 22 uir decorus (passim)

cunctis uidentibus $3,63,16$

superaltare posuit $2,11,10$;

$4,111,22$

magno opere $4,126,42$

circumstantes $4,86,23$

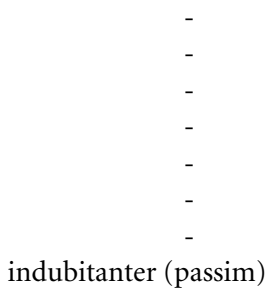

forma perspicuae uitae $0,2,8$ dux memoralis uitae $0,7,22$ uiros honestissimae uitae 3 , 36,8

missarum solemnis $1,7,1$ more solito 2, 5, 15 / 2, 23, 3

mysterio celebrato $4,121,59$ quae accederant referre 2, 24, 54 ;

4, 99, 39

repletus uirtute / stultitia...

gratias agere 2, 19, 28

gratias referre $4,119,4$

congregata multitudine $2,3,3$; 2, 9, 5 / 3, 51, 19

dux, supra memoratus 3, 61, 6 conuocatis prrincipibus (7: passim)

magno gaudio $3,58,3$ 